\title{
An Electro-hydrodynamics-based Model Studying Features of Electroosmotic Flow in a Solid-state Nanopore
}

\author{
W.Y. Zhao \\ State Key Laboratory of Bioelectronics \\ Southeast University \\ Nanjing, China \\ J.C. Li \\ State Key Laboratory of Bioelectronics \\ Southeast University \\ Nanjing, China \\ R.C. $\mathrm{Ke}$ \\ State Key Laboratory of Bioelectronics \\ Southeast University \\ Nanjing, China
}

\author{
L.M. Lu \\ State Key Laboratory of Bioelectronics \\ Southeast University \\ Nanjing, China \\ Q.J. Liu \\ State Key Laboratory of Bioelectronics \\ Southeast University \\ Nanjing, China \\ Z.H. Lu \\ State Key Laboratory of Bioelectronics \\ Southeast University \\ Nanjing, China
}

\begin{abstract}
Nanopore technology for single molecule detection has a great potential in DNA sequencing and other bio-analytical applications. The magnitude of current signals can reach subnano-ampere level, the stability of signals and signals to noise ratio are very important in single molecule detection. In this work, a steady-state continuum-based model, composed of the coupled Poisson-Nernst-Planck equations was used for investigating the electroosmotic flow (EOF) inside the nanopore. The features of EOF were figured and the changes with the variables such as ion concentration, pore size were analyzed. A flat profile of EOF was discovered and some effective factors for inducing this kind flow were researched. Understanding the origin and the nature of this EOF profile especially to nanopore geometry is of great significance to develop solid-state nanopore technology. This may help to control the kinestate of nanoparticles inside the pore, analyze and discriminate those current signals, and even improve the stability of detecting signals.
\end{abstract}

\section{Keywords- nanopore; PNP equation; EOF; current signals}

\section{INTRODUCTION}

Nanopore sensor device has been developed as an effective platform for individual molecule detection. Solid-state nanopores with advantages as flexible diameters and membrane thickness, and steady chemical properties and the possibility for controllable fabrication process were proposed as an alternative detector in this field [1-3]. The detecting signals are reflection of ionic current response due to the nanoparticles translocating through nanopore. Particles or bio-molecules are electrophoretically driven through a single nanopore by an external electric field, this will give rise to a change of the ionic conductivity inside the nanopore $[4,5]$. From signals recording these changes, particles' physical feathers (such as the size and charge) and dynamic properties (such as mobility and capture rate) can be inferred [6-8]. Experimental studies have found that ionic current response depends on several factors ,such as the externally applied electric field, the ionic concentration [9], the nanopore size, and the shape of the nanoparticles [7, 10-12]. Furthermore, modeling and simulations are also made to a more detailed understanding of the particle translocation through a nanopore $[4,13,14]$. Actually, solid-state nanopore is usually charged and surrounded by electrolyte solution, ion concentration polarization and counterions accumulate in a thin liquid layer adjacent to the nanopore and membrane which is known as EDL. Those excess counterions will be driven by external electrical field and thus follows the fluid which leads to electroosmotic flow (EOF). As the magnitude of EOF could almost complete with that of electrophoresis, and always present an opposite direction, this flow can induce a hydrodynamic drag force on particles that locally balances the electrical force [7, 15-17]. Over all, the drag force induced by EOF contributes a strong impact on particle dynamics inside the pore which cannot be ignored. Besides, along all the factors, the induced EOF is prone to be alterable under different 
conditions, for instance, when the nanopore size, shape surface charge and ionic concentration are changed [18].

In this study, EOF inside a solid-state nanopore was investigated using a continuum model, composed of the coupled Poisson-Nernst-Planck equations for the ionic mass transport and Navier-Stokes equations for the hydrodynamic field [4, 14, 18]. The EOF profiles inside nanopore were figured and some affective factors, such as nanopore size and ion concentration were explored. As the magnitude of the current signal can reach sub-nano-ampere, the kinestate of particles inside the pore will cause an obvious impact on those signals [19]. In addition, the non-uniform EOF will have great effects on translocation process, especially for rod-like and spherical particles. Understanding the characteristics of EOF may help to analyze and discriminate those current signals, and is also helpful to design more reasonable nanopore device.

\section{METHOD}

\section{A. Mathematical Model}

Our nanopore system is built by drilling a cylindrical pore of radius $b$ on a membrane of thickness $L$. The nanopore is connected to two identical reservoirs of width $2 \mathrm{~W}$ and height $\mathrm{H}$ filled with a binary $\mathrm{KCl}$ aqueous solution. A sketch of the nanopore system is shown in fig.1. Actually, the two reservoirs are large enough to maintain a bulk ionic concentration $\mathrm{C}_{0}$ that far away from the nanopore. As we use an axisymmetric model here, all the variables are defined in a cylindrical coordinate system $(\mathrm{r}, \mathrm{z})$ with the origin fixed at the center of the nanopore. An electric field $\mathrm{E}$ is induced across the nanopore by applying an external potential $\mathrm{V}_{0}$ across two electrodes positioned inside the two reservoirs. The nanopore and membrane are negatively charged, with a fixed surface charge density $\mathrm{c}_{w}$, and ignore other charges. All the constants and variables are listed as follows: $c_{i}(i=1,2)$ is the molar concentration of cation $\left(\mathrm{K}^{+}\right)$and anion $\left(\mathrm{Cl}^{-}\right)$, density, dynamic viscosity and permittivity of the electrolyte solution were $\rho, \mu$ and $\varepsilon_{f}$ respectively. The diffusivity and valence of the $i$ th species are $\mathrm{D}_{i}$ and $z_{i}\left(z_{1}=1\right.$ and $z_{2}=-1$ for $\mathrm{KCl}$ ); $\mathrm{R}$ is the universal gas constant; $\mathrm{T}$ is the absolute temperature set $300 \mathrm{~K}$ here; $\varepsilon_{0}$ is the absolute permittivity of the vacuum; is the electric potential within the fluid; $F$ is the Faraday constant.

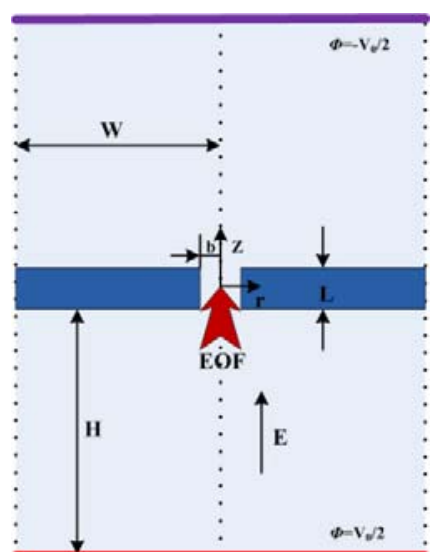

FIGURE I. HE SCHEMATIC OF NANOPORE SYSTEM (2DSYMMETRIC).
The nanopore system is running in a stationary state, for incompressible fluid the solution domain consists of continuity equation:

$$
\nabla \cdot \mathbf{u}=0
$$

Since the Reynolds number of electrokinetic flow in the nanopore is extremely small, the modified Navier-Stokes equations by neglecting the inertial terms are used here:

$$
\rho \frac{\partial \mathrm{u}}{\partial t}=-\nabla p+\mu \nabla^{2} \mathbf{u}-\mathrm{F}\left(z_{1} c_{1}+z_{2} c_{2}\right) \nabla \phi
$$

Poisson equation and Nernst-Planck equations are used to describe electric potential and ionic mass transport.

$$
\begin{gathered}
-\varepsilon_{0} \varepsilon_{f} \nabla^{2} \phi=\mathrm{F}\left(c_{1} z_{1}+c_{2} z_{2}\right) \\
\frac{\partial c_{i}}{\partial t}+\nabla \mathrm{N}_{i}=0, i=1,2 \\
\mathrm{~N}_{i}=\mathrm{u} c_{i}-D_{i} \nabla c_{i}-z_{i} \frac{D_{i}}{R T} F c_{i} \nabla \phi
\end{gathered}
$$

The boundary conditions for the nanopore system are as follows: ionic concentrations at the ends of the two reservoirs are $c_{i}(\mathrm{x}, \pm(\mathrm{H}+\mathrm{h} / 2))=\mathrm{C}_{0}, i=1$ and 2 ; other boundaries all are set to be insulation/symmetry for $c_{i}$. The electric potential applied at the ends of the two reservoirs are $(\mathrm{x},-(\mathrm{H}+\mathrm{h} / 2))=\mathrm{V}_{0} / 2, \quad(\mathrm{x}$, $(\mathrm{H}+\mathrm{h} / 2))=-\mathrm{V}_{0} / 2$, the nanopore wall and membrane surface charge $\rho_{\mathrm{s}}=\sigma_{\mathrm{w}}$ with equation $-\mathbf{n} \cdot \varepsilon_{0} \varepsilon_{f} \nabla=\sigma_{\mathrm{w}}$, other boundaries are zero charge/symmetry and equation is $\mathbf{n}$. $\varepsilon_{0} \varepsilon_{f} \nabla=0, \mathbf{n}$ is the unit normal vector. For solving fluid flow field, the nanopore and the membrane boundary are set as wall/no-slip, with equation as $\mathbf{u}=0$; reservoir walls are set as wall/symmetry, the equations are $\mathbf{n} \cdot \mathbf{u}=0, \mathbf{t} \cdot\{[-\mathbf{p} \cdot \mathbf{I}+$ $\left.\left.\left(\nabla \mathbf{u}+\nabla \mathbf{u}^{\mathrm{T}}\right)\right] \cdot \mathbf{n}\right\}=0, \mathrm{I}=\int \mathrm{F}\left(z_{1} \cdot \mathbf{N}_{\mathbf{1}}+z_{2} \cdot \mathbf{N}_{\mathbf{2}}\right) \cdot \mathbf{n d} \mathbf{S} ;$ with $\mathbf{t}$ denoting the unit tangential vector on the boundary; the two ends of the nanopore condition are outlet/pressure, no viscous stress $\mathrm{P}_{0}=0$, with equation of $u\left(\nabla \mathbf{u}+\nabla \mathbf{u}^{\mathrm{T}}\right) \mathrm{n}=\mathbf{0}, \mathrm{P}=\mathrm{P}_{0}$.

\section{B. Dimensionless Simulation}

In order to facilitate the calculation, we present the dimensionless form of the equations and physical quantities to govern the physics behavior. Here, the bulk concentration $c_{0}$ is the scale of $c_{i}$; electric potential is scaled with $\mathrm{R} \cdot \mathrm{T} / \mathrm{F}, a$ is the length scale, $\mathrm{U}_{0}=\varepsilon_{0} \varepsilon_{f} \mathrm{R}^{2} \mathrm{~T}^{2} /\left(u a \mathrm{~F}^{2}\right)$ is the velocity scale, and $u \mathrm{U}_{0} / a$ is the pressure scale. All these scale are used to normalize the governing equations.

\section{Numerical Implementation}

The finite element package COMSOL MULTIPHYSICS is used to solve the PNP equation system numerically. The computational domain is discretized into quadratic triangular elements, especially the nanopore wall and the membrane surface with a higher element density to resolve the adjacent EDLs. Here, $\mathrm{W}$ and $\mathrm{H}$ is much larger than nanopore size, $\mathrm{L}$ and $\mathrm{b}$ are used with different value. Some parameters used in the simulations are listed as follows: D1 $=1.95 \times 10^{-9} \mathrm{~m}^{2} / \mathrm{s}, \mathrm{D} 2=2.03$ $\times 10^{-9} \mathrm{~m}^{2} / \mathrm{s} ; \quad \mathrm{F}=96485.3415 \mathrm{C} / \mathrm{mol}, \quad \mathrm{R}=8.314472 \mathrm{~J} / \mathrm{K} / \mathrm{mol}$, $\mathrm{T}=300 \mathrm{~K}, \quad \varepsilon_{0}=8.8542 \times 10^{-12} \mathrm{~F} / \mathrm{m}, \quad \varepsilon_{f}=80, \mu=8.9 \times 10^{-9} \mathrm{pa} \cdot \mathrm{s}$, $\mathrm{a}=1 \times 10^{-9} \mathrm{~m}, \rho=1 \times 10^{3} \mathrm{~kg} / \mathrm{m}^{3}$. 


\section{RESULT AND DISCUSSION}

The EOF of the nanopore system mainly contains two types, one is circulating in two reservoirs respectively, the other is inside the nanopore channel. Since the effect of concentration polarization and EDLs inside the nanopore, the latter type always exhibits a larger magnitude value, and then imposes a significant effect on particle translocation. Fig. 2 shows the distribution of electric field and flow field in the nanopore system. In present study, electric field screens the surface charge of EDLs in motion which drag the flow, the velocity of EOF inside the nanopore is proportional to the electric field applied to the pore and the surface charge of the wall. A equation of the electroosmotic velocity is proposed when the Debye length is much smaller than the channel width [15]. $\mathrm{V}_{\mathrm{EO}}=-\frac{\varepsilon_{0} \varepsilon_{f}}{\mu} \xi_{\text {wall }} E$.

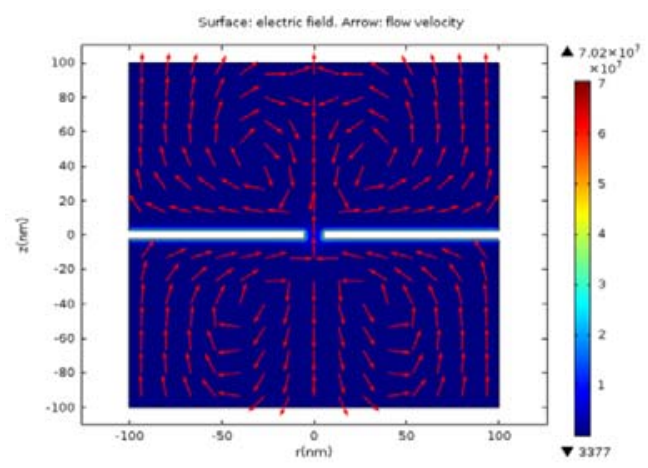

FIGURE II. DISTRIBUTION OF ELECTRIC AND FLOW FIELD (NORMALIZED). THE ELECTRIC FIELD VALUE OF COLOR BAR WERE IN INTERNATIONAL STANDARD UNIT V/M.C $0=0.1 \mathrm{M}$, $\mathrm{L}=\mathrm{B}=5 \mathrm{NM}$, VOLTAGE BIAS IS SET AS 100MV, $\Sigma \mathrm{W}=-0.036 \mathrm{C} / \mathrm{M} 2$.

The EOF profiles in the nanopore were presented in fig. 3 (a), and fig. 3 (b) reflects the gap of flow velocity value in the nanopore system. With the varying of bulk concentration $\mathrm{C}_{0}$ in a fixed nanopore system, the shape and value size of EOF inside the pore will exhibit a significant change; see fig. 3 (c). Since the Debye length $\kappa^{-1}=\sqrt{\varepsilon_{0} \varepsilon_{\mathrm{f}} \mathrm{R} \cdot \mathrm{T} / \sum_{\mathrm{i}=1}^{2} \mathrm{~F}^{2} \mathrm{z}_{\mathrm{i}}^{2} \mathrm{C}_{0}}$ is relate to $\mathrm{C}_{0}$, this will cause a change of the distribution of net ionic concentration, and so is the flow feature. For a fixed nanopore, there exist two threshold concentrations, with one can lead to a maximum peak of flow velocity, with the other will cause a uniform EOF. This can help us choose a proper bulk concentration to control the effect of electroosmotic flow on nanoparticles. The uniform EOF is in favor of nanoparticles bearing uniform forces, which is easy for maintaining particles' behavior stable and consistent when translocating through the nanopore. In addition, under a constant bulk concentration and electric field, when changing the size of nanopore, such as the ratio of aperture and membrane thickness, the EOF profile will also exhibit a change from concavity to convexity. Of course, there must exist a uniform EOF under a corresponding value of aperture and thickness. fig. 3 (d) shows the change of EOF profile with a fixed aperture at $4 \mathrm{~nm}$ and changing the membrane thickness from $5 \mathrm{~nm}$ to $100 \mathrm{~nm}$. Defining the uniform EOF as the difference of most flow values along the $r$ - coordinate were the smallest (at least less than $0.001 \mathrm{~m} / \mathrm{s}$ ), these flow sites locate at most of the center region inside the nanopore, such as the blue line $(\mathrm{L}=20 \mathrm{~nm}, \mathrm{U}=0.4 \mathrm{~V})$ in fig. 3 (d). Some proper apertures (D) and thickness (L) for inducing the uniform EOF is figured at fig. 4. Those proper apertures and thickness are fitted by an exponential function, $L=-8.947+15.217 e^{\frac{D-3.157}{1.465}}$. Noted, for $1 \mathrm{~mol} / \mathrm{L}$ electrolyte concentration, the profile of EOF will keep in a convex form when the aperture of nanopore less than $3 \mathrm{~nm}$, and with the increase of aperture, there emerge an exponential rise of the thickness for inducing the uniform EOF. The uniform EOF is good for particle translocation in a stable state with fewer collisions with the nanopore wall, especially for rod-like particles, which are easily titled by the flow. Under this uniform EOF, we can easily obtain those more stable current signals, with less fluctuation.

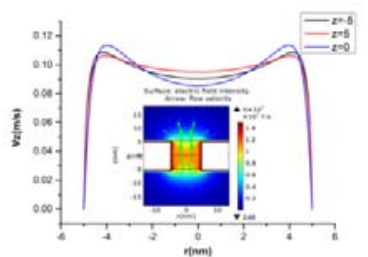

(a)

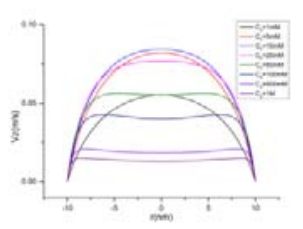

(c)

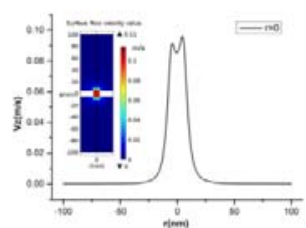

(b)

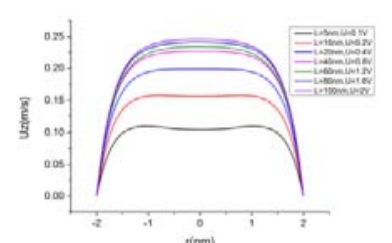

(d)
FIGURE III. (A) THE FLOW VELOCITY VALUE DISTRIBUTION ALONG THE LATERAL AXIS $Z=0, Z=5, Z=-5$, THE GREEN ARROW REPRESENT FLOW VELOCITY PROPORTIONALLY. (B) THE FLOW VELOCITY VALUE DISTRIBUTION ALONG THE VERTICAL AXIS $\mathrm{R}=0$. $\mathrm{C} 0=1 \mathrm{M}, \mathrm{L}=\mathrm{B}=10 \mathrm{NM}, \mathrm{VOLTAGE}$ BIAS IS SET AS $200 \mathrm{MV}, \Sigma \mathrm{W}=$ $0.036 \mathrm{C} / \mathrm{M} 2, \mathrm{BOTH}$ IN (A) AND (B). (C) THE PROFILE OF EOF WITH THE VARYING OF BULK CONCENTRATION CO.

$\mathrm{B}=10 \mathrm{NM}, \mathrm{L}=50 \mathrm{NM}, \mathrm{V} 0=200 \mathrm{MV}, \Sigma \mathrm{W}=0.015 \mathrm{C} / \mathrm{M} 2$. (D) THE PROFILE OF EOF WITH THE CHANGING OF L, THE ELECTRIC FIELD STRENGTH WAS KEPT AS A CONSISTENT VALUE. $\mathrm{B}=2 \mathrm{NM}, \mathrm{E}=2 \times 107 \mathrm{~V} / \mathrm{M}$.

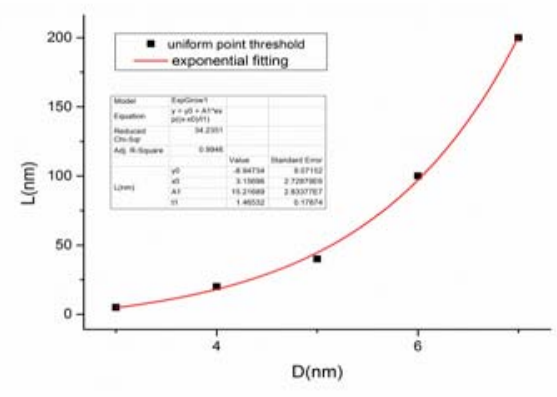

FIGURE IV. THE PROPER APERTURE (L) AND THICKNESS ( $D=2 \mathrm{~B})$ FOR INDUCING UNIFORM EOF WHEN ELECTRIC FIELD WAS KEPT AS $2 \times 107 \mathrm{~V} / \mathrm{M}$. 


\section{CONCLUSION}

In summary, using a numerical simulation method, we depicted the feature of EOF inside the nanopore, and we analyzed the changing features of the flow profile with the varying of ion concentration and the pore size. For a fixed nanopore, there exists a special bulk concentration with which can lead to a maximum peak of flow velocity or flat EOF profile, and this phenomenon will be deeply explored in our further studies. Besides, there exists a proper corresponding value of pore aperture and membrane thickness, which can also lead to a flat flow profile. This uniform EOF is good for particle translocation in a stable state with fewer collisions with the nanopore wall, and then the detected signals are more likely to be stable and easy to discrimination. In further study, we will systematically to dig out those threshold conditions under different pore size and bulk concentration and even other unknown effective factors. This is useful and significant for developing nanopore technology.

\section{ACKNOWLEDGMENTS}

The work was supported by the National Basic Research Program of China (2011CB707600), the National Natural Science Foundation of China $(61071050,61372031)$, and Tsinghua National Laboratory for Information Science and Technology (TNList) Cross-discipline Foundation.

\section{REFERENCES}

[1] Wanunu, M. Nanopores: A journey towards DNA sequencing. Physics of Life Reviews. 9(2):p.125-158.2012

[2] Schneider, G.F. and C. Dekker DNA sequencing with nanopores. Nature Biotechnology. 30(4):p.326-328.2012

[3] Healy, K.B. Schiedtand A.P. Morrison Solid-state nanopore technologies for nanopore-based DNA analysis. Nanomedicine. 2(6):p.875-897.2007

[4] Ai, Y. and S.Z. Qian Electrokinetic particle translocation through a nanopore. Physical Chemistry Chemical Physics. 13(9):p.40604071.2011

[5] Kesselheim, S.W. Mullerand C. Holm Origin of Current Blockades in Nanopore Translocation Experiments. Physical Review Letters. 112(1).2014

[6] Aksimentiev, A. Deciphering ionic current signatures of DNA transport through a nanopore. Nanoscale. 2(4):p.468-483.2010

[7] Luan, B. and G. Stolovitzky An electro-hydrodynamics-based model for the ionic conductivity of solid-state nanopores during DNA translocation. Nanotechnology. 24(19):p.195702.2013

[8] de Haan, H.W. and G.W. Slater Translocation of "Rod-Coil" Polymers Probing the Structure of Single Molecules within Nanopores. Physical Review Letters. 110(4).2013

[9] Ghosal, S. Effect of salt concentration on the electrophoretic speed of a polyelectrolyte through a nanopore. Physical Review Letters. 98(23). 2007

[10] Meller, A. and D. Branton Single molecule measurements of DNA transport through a nanopore. Electrophoresis. 23(16):p.2583-2591.2002

[11] Smeets, R.M. et al. Salt dependence of ion transport and DNA translocation through solid-state nanopores. Nano Lett. 6(1):p.8995.2006

[12] Lee, C. et al. Large apparent electric size of solid-state nanopores due to spatially extended surface conduction. Nano Lett. 12(8):p.40374044.2012
[13] Ai, Y. and S. Qian Direct numerical simulation of electrokinetic translocation of a cylindrical particle through a nanopore using a Poisson-Boltzmann approach. Electrophoresis. 32(9):p.996-1005.2011

[14] Ai, Y. et al. Field effect regulation of DNA translocation through a nanopore. Anal Chem. 82(19):p.8217-8225.2010

[15] Firnkes, M. et al. Electrically Facilitated Translocations of Proteins through Silicon Nitride Nanopores: Conjoint and Competitive Action of Diffusion, Electrophoresis, and Electroosmosis. Nano Lett. 10(6):p.2162-2167.2010

[16] Lo, T.-W. et al. Diffusiophoresis of a Charged Sphere in a Necked Nanopore. The Journal of Physical Chemistry C. 117(37):p.1922619233.2013

[17] Mao, M.S. Ghosaland G.H. Hu Hydrodynamic flow in the vicinity of a nanopore induced by an applied voltage. Nanotechnology. 24(24).2013

[18] Wong, C.T.A. and M. Muthukumar Polymer capture by electro-osmotic flow of oppositely charged nanopores. Journal of Chemical Physics. 126(16).2007

[19] $\mathrm{Wu}$, L.Z. et al. Electrically facilitated translocation of protein through solid nanopore. Nanoscale Research Letters. 9.2014 